\title{
Salvianolic Acid A Protects Against Diabetic Nephropathy through Ameliorating Glomerular Endothelial Dysfunction via Inhibiting AGE-RAGE Signaling
}

Biyu Hou ${ }^{\mathrm{a}}$ Guifen Qiang ${ }^{\mathrm{a}}$ Yuerong Zhao ${ }^{\mathrm{a}, \mathrm{b}} \quad$ Xiuying Yang ${ }^{\mathrm{a}} \quad$ Xi Chen ${ }^{\mathrm{a}} \quad$ Yu Yan ${ }^{\mathrm{a}}$ Xiaobo Wang ${ }^{\mathrm{a}, \mathrm{b}}$ Chenge Liu ${ }^{\mathrm{a}}$ Li Zhang $^{\mathrm{a}}$ Guanhua Du ${ }^{\mathrm{a}}$

aBeijing Key Laboratory of Drug Target Identification and Drug Screening, Institute of Materia Medica,

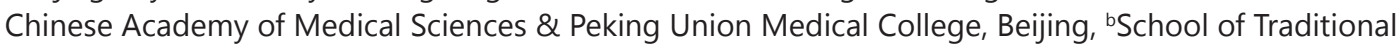
Chinese Medicine, Guangdong Pharmaceutical University, Guangzhou, China

\section{Key Words}

Salvianolic acid A - Advanced glycation end products $\cdot$ Diabetic nephropathy $\cdot$ Glomerular endothelia dysfunction.

\begin{abstract}
Background/Aims: Glomerular endothelium dysfunction leads to the progression of renal architectonic and functional abnormalities in early-stage diabetic nephropathy (DN). Advanced glycation end products (AGEs) and receptor for AGEs (RAGE) are proved to play important roles in diabetic nephropathy. This study investigated the role of Salvianolic acid A (SalA) on early-stage DN and its possible underlying mechanism. Methods: In vitro AGEs formation and breaking rate were measured to illustrate the effect of SalA on AGEs. Type 2 diabetic nephropathy rats were induced by high-fat diet and low-dose streptozocin (STZ). After eight-week treatment with SalA $1 \mathrm{mg} / \mathrm{kg} /$ day, 24h-urine protein, creatinine clearance was tested and renal structural injury was assessed by PAS and PASM staining. Primary glomerular endothelial cell permeability was evaluated after exposed to AGEs. AGEs-induced RhoA/ROCK and subsequently activated disarrange of cytoskeleton were assessed by western blot and immunofluorescence. Results: Biochemical assay and histological examination demonstrated that SalA markedly reduced endothelium loss and glomerular hyperfiltration, suppressed glomerular hypertrophy and mesangial matrix expansion, eventually reduced urinary albumin and ameliorated renal function. Further investigation suggested that SalA exerted its renoprotective effects through inhibiting AGE-RAGE signaling. It not only inhibited formation of AGEs and increased its breaking in vitro, but also reduced AGEs accumulation in vivo and downregulated RAGE expression. SalA restored glomerular endothelial permeability through suppressing AGEs-induced rearrangement of actin cytoskeleton via AGE-RAGE-RhoA/ ROCK pathway. Moreover, SalA attenuated oxidative stress induced by AGEs, subsequently alleviated inflammation and restored the disturbed autophagy in glomerular endothelial cell and diabetic rats via AGE-RAGE-Nox4 axis. Conclusion: Our study indicated that SalA restored glomerular endothelial function and alleviated renal structural deterioration through inhibiting

Li Zhang

and Guanhua Du

Institute of Materia Medica, Chinese Academy of Medical Sciences \&

Peking Union Medical College, Beijing (China)

E-Mail zhangli@imm.ac.cn, dugh@imm.ac.cn
\end{abstract}


AGE-RAGE, thus effectively ameliorated early-stage diabetic nephropathy. SalA might be a promising therapeutic agent for the treatment of diabetic nephropathy.

(C) 2017 The Author(s)

Published by S. Karger AG, Basel

\section{Introduction}

Diabetic nephropathy (DN) is one of the most common complications of diabetes and the leading cause of end-stage renal disease. Glomerular hyperfiltration is notable in earlystage DN since it causes albuminuria and initiates the sclerotic process of glomeruli, resulting in fibrosis and irreversible renal failure [1,2]. Growing evidence suggests that endothelial dysfunction is the central link in increasing permeability of glomerular filtration barrier [3]. Endothelial injury is manifested through loss of extracellular glycocalyx [4], endothelial hyperpermeability [5], disrupted secretion of endothelial-derived vasoactive mediators, and phenotype changes [6].

The accumulation of AGEs plays a crucial part in the onset and progress of DN. AGEs exert effect through cross-link directly or by binding various receptors on cell surface such as receptor for AGEs (RAGE) indirectly [7]. These events stimulate the production of aroused reactive oxygen species (ROS) and ultimately lead to increased vascular permeability and inflammation. Reports showed that disarrangement of F-actin cytoskeleton induced by activation of RhoA/ROCK signaling is the main mechanism in AGEs-RAGE injured endothelial cell hyperpermeability [8]. Moreover, AGE-RAGE initiates the cascades of signaling transduction such as AGE-RAGE-NADPH oxidase (Nox) to aggravate oxidative stress [9], or AGE-RAGE-Jak/Stat to interfere the biosynthesis of collagen in kidney fibroblast [10]. These downstream pathways are involved widely in inflammation, autophagy, and apoptosis exerting an extend influence on cellular metabolism. Thus, inhibition of the AGEs formation and/or the blockade of RAGE-downstream pathways have become a promising therapeutic strategy for DN.

Salvianolic acid A (SalA), ((2R)-3-(3, 4-dihydroxyphenyl)-2-[(E)-3-[2-[(E)-2-(3, 4-dihydroxyphenyl) ethenyl]-3, 4-dihydroxyphenyl] prop-2-enoyl] oxypropanoic acid) is a water-soluble phenolic acid extracted from the dried root and rhizome of Salvia miltiorrhiza Bunge [11]. Studies demonstrated that SalA has various pharmacological effects such as anti-ischemia [12], anti-inflammation [13] and anti-diabetic [14]. Especially, SalA displays a beneficial effect on diabetic complications, including hepatic fibrosis [15], diabetic vascular endothelial dysfunction [16] and diabetic peripheral neuropathy [17]. All findings indicate that SalA has multiple protective effects on diabetic macrovascular and microvascular complications. In addition, Fan [18] reported that SalA exerted renal protection on doxorubicin-induced nephropathy through ameliorating podocyte injury and suppressing oxidative stress and inflammation. However, the effect of SalA on diabetic nephropathy, especially the glomerular endothelial hyperpermeability remains unknown yet. According to reviews of SalA on diabetic complications, we speculated that SalA could play a protective role in diabetic nephropathy.

In this study, we found that SalA protected against early-stage diabetic nephropathy by ameliorating glomerular endothelial hyperpermeability in type 2 diabetic rats. Furthermore, we first demonstrated that SalA played its renoprotective effects through AGE-RAGE-NADPH oxidase 4 (Nox4) and AGE-RAGE-RhoA/ROCK signaling pathway.

\section{Materials and Methods}

\section{Reagents and Antibodies}

SalA (HPLC, 98\%) was provided as a lyophilized powder by the Institute of Materia Medica, Chinese Academy of Medical Sciences (Beijing, China). Streptozocin (STZ) was purchased from Sigma Chemical Co, USA. Advanced glycation end products (AGEs), was obtained from Bioss Chemical, China. All other chemicals are of analytical grade. The antibodies used in our study were as follows: rabbit anti-RAGE (Cat. ab3611),

\section{KARGER}


rabbit anti-Nox4 (Cat. ab109225), rabbit anti-Interleukin-6 (IL-6) (Cat. ab6672), rabbit anti-Tumor Necrosis Factor- $\alpha$ (TNF- $\alpha$ ) (Cat. ab6671) (Abcam, USA), mouse anti-Aminopeptidase P (JG-12) (Cat. sc-65390), rabbit anti-Interleukin-1 $\beta$ (IL-1 $\beta$ ) (Cat. sc-7884), mouse anti-Intercellular Adhesion Molecule 1 (ICAM-1) (Cat. sc8439), mouse anti-Vascular Cell Adhesion Molecule-1 (VCAM-1) (Cat. sc13160) (Santa cruz, USA), rabbit anti-RhoA (Cat. 2117), rabbit anti-ROCK (Cat. 9029), rabbit anti-Phospho-Myosin Light Chain (p-MLC) (Cat. 3671), rabbit anti-Myosin Light Chain (t-MLC) (Cat. 3672), rabbit anti-p62/SQSTM1 (Cat. 5114), rabbit antiLC3A/B (Cat. 12741), rabbit anti-Bclin-1 (Cat. 3495), rabbit anti-Sirt1 (Cat. 9475), rabbit anti-Forkhead Transcription Factors-3a (Foxo3a) (Cat. 12829s), rabbit anti-BCL2/Adenovirus E1B 19 kDa ProteinInteracting Protein 3 (Bnip3) (Cat. 12396s), rabbit anti-Atg5 (Cat. 12994), rabbit anti-Atg7 (Cat. 8558), rabbit anti-Atg12 (Cat. 4180) (CST, USA), mouse anti- $\beta$-Actin (Cat. 3010ES50) (Yeasen, China).

\section{AGEs Measurement}

Antiglycation activity of SalA in vitro was tested by the ability to inhibit the fluorescence of AGEs as described in previous study [19] with a minor modification. Briefly, a reaction mixture of $5 \mathrm{mg} / \mathrm{ml}$ bovine serum albumin (BSA) , $0.5 \mathrm{M}$ glucose, $1 \%$ glyoxal, $0.1 \mathrm{mM} \mathrm{PMSF}, 100 \mathrm{U} / \mathrm{ml}$ penicillin and $100 \mathrm{U} /$ $\mathrm{ml}$ streptomycin in $0.1 \mathrm{M}$ phosphate buffered-saline (PBS), pH 7.4 with different concentrations of SalA was incubated in darkness at $37{ }^{\circ} \mathrm{C}$ for 4 days. AGEs formation was assessed by fluorescent intensity at excitation wavelength of $355 \mathrm{~nm}$ and emission wavelength of $460 \mathrm{~nm}$. The AGEs cross-link breaking activity of SalA was assessed by monitoring the fluorescent intensity change of AGEs after incubated with different concentrations of SalA for 4 days. The remained AGEs were estimated by fluorescent intensity at excitation wavelength of $355 \mathrm{~nm}$ and emission wavelength of $460 \mathrm{~nm}$.

\section{Animals Experimental Design}

Male Sprague-Dawley (SD) rats (140-160 g) purchased from Beijing HFK Bioscience Co. Ltd (Beijing, China) were kept under a SPF environment $\left(22-25{ }^{\circ} \mathrm{C}\right.$, humidity $60-70 \%, 12$-h light/dark cycle) for one week to acclimate these conditions. All animals were handled in accordance with ethical guidelines for using and caring of laboratory animals with the permission of animal care committee of the Institute of Materia Medica, Chinese Academy of Medical Sciences.

The rat model of diabetic nephropathy was induced as previously described [20] with a minor modification (Fig.1). In brief, rats were fed with a high-fat and high-sucrose diet (standard diet supplemented with $10 \%$ sucrose, $10 \%$ lard stearin, $2 \%$ cholesterin, and $0.5 \%$ cholic acid) for four weeks. After fasted overnight, $35 \mathrm{mg} / \mathrm{kg} \mathrm{STZ}$ was administered by a single intraperitoneal injection after dissolved with $0.1 \mathrm{M}$ citrate buffer ( $\mathrm{pH}$ 4.4), while normal control animals received only citrate buffer. Rats with fasting blood glucose (FBG) higher than $10 \mathrm{mM}$ and lower than $25 \mathrm{mM}$ were considered as diabetes, and then fed with HFD (10\% lard stearin, $2 \%$ cholesterin and $0.5 \%$ cholic acid) for another eight weeks. Meanwhile, diabetic rats were randomly assigned into two groups: diabetic model rats (DM), and diabetic rats with orally administration of SalA at $1 \mathrm{mg} / \mathrm{kg} / \mathrm{day}$ (SalA). Age-matched rats were served as normal control and were treated with vehicle and standard chow diet during the entire experiment. At the end of the eight-week treatment, rats were sacrificed after fasting overnight. Blood samples were collected and kidney tissue was immediately frozen in liquid nitrogen or fixed in $4 \%$ paraformaldehyde for histological diagnosis.

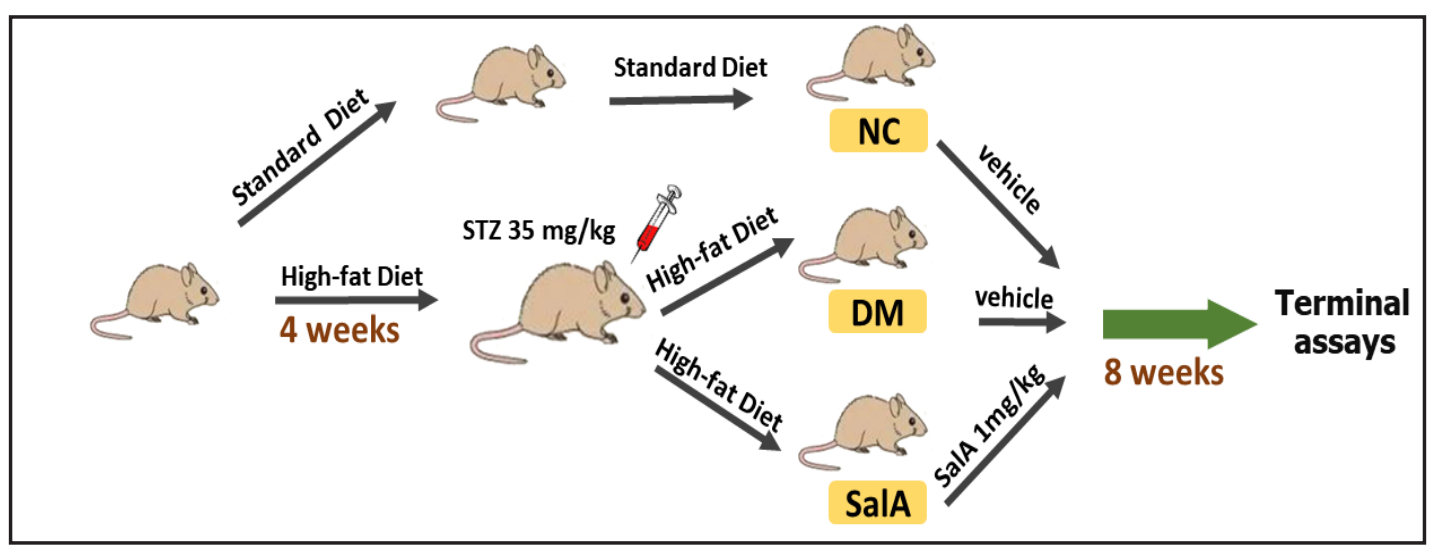

Fig. 1. Animal experiment flow. 


\section{Cellular Physiology Cell Physiol Biochem 2017;44:2378-2394 \\ \begin{tabular}{ll|l} 
and Biochemistry Published online: December 18, 2017 & $\begin{array}{l}\text { C) } 2017 \text { The Author(s). Published by S. Karger AG, Basel } \\
\text { www.karger.com/cpb }\end{array}$ \\
\hline
\end{tabular}}

Hou et al.: Renoprotective Effects of Salvianolic Acid A

\section{Plasma and Urine Biochemical Determination}

Fasting blood glucose levels from tail-vein were measured by ACCU-CHEK® active glucometer (Roche, Hoffmann, Germany) at $2^{\text {nd }}, 4^{\text {th }}, 6^{\text {th }}$, and $8^{\text {th }}$ week of administration. Blood lipids of total cholesterol (TCHO), triglycerides (TG), high density lipoprotein (HDL), low density lipoprotein (LDL) were detected with an automatic analyzer (TOSHIBA Acute TBA-40FR, TOSHIBA CORPORATION, Tokyo, Japan). Serum level of glycation serum protein (GSP), creatinine (Scr) and blood urea nitrogen (BUN) were assessed according to the manufacturer's instructions by standard diagnostic kits (Jiancheng Biotech Co. Ltd. Nanjing, China).

The specific markers for oxidative stress including serum level of malondialdehyde (MDA) and the activity of superoxide dismutase (SOD) were analyzed by commercial kits (Jiancheng Biotech Co., Ltd., Nanjing, China). Serum level of nitric oxide (NO) was assayed by Griess reagent and renal cortex catalase (CAT) activity was performed using catalase assay kit following manufacturer's instruction (Beyotime Institute of Biotechnology, Shanghai, China). Serum level of 8-OHdG and concentration of AGEs in serum and renal cortex were conducted as the manufacturer's instructions using a commercially available kit (CUSABIO, Wuhan, China).

Urine sample collections were conducted referred to previous report [21]. At $8^{\text {th }}$ week, rats were held in metabolism cages for 24 hours with access to food and water. $24 \mathrm{~h}$-urine volume was recorded and urinary albumin concentration was measured by ELISA assay (CUSABIO, Wuhan, China). Urinary creatinine concentrations were measured using a creatinine assay kit (Jiancheng Biotech Co., Ltd., Nanjing, China). Creatinine clearance $(\mathrm{Ccr})$ was calculated using the following formula: $\mathrm{Ccr}(\mu \mathrm{l} / \mathrm{min})=(\mathrm{Ucr} / \mathrm{Pcr}) \times$ urine volume ( $\mu \mathrm{l} / \mathrm{min})$, as P. Palsamy et al. reported [22]. Albumin creatinine ratio (UACR) was expressed as the ratio of total urinary albumin to creatinine, as previously described [23].

\section{Histopathology Analysis and Immunohistochemistry Staining}

Morphological Analysis

Kidney mesangial expansion was assessed referred to previous study [24]. Fifteen glomeruli randomly selected from each rat were measured. The Periodic Acid-Silver Methenamine (PASM)-positive material in the mesangial area and glomerular tuft area was calculated by Image J 4.5 software (NIH, USA). Periodic acid Schiff-stained was carried out to evaluate the glomerular volume [25]. Briefly, the mean glomerular tuft volume (GV) was determined of the mean glomerular cross-sectional area (GA) under optical microscopy. GA was estimated by the average area of a total of fifteen cortical glomeruli from each animal. GV was calculated as $\mathrm{GV}=\beta / \kappa \times(\mathrm{GA}) 3 / 2$, where $\beta=1.38$, which is the shape coefficient for spheres (the idealized shape of glomeruli), and $\kappa=1.1$, which is a size distribution coefficient. HE staining was conducted and renal interstitial inflammation was scored as previously reported [26]. Briefly, inflammation was scored on a range of 0 to 3. 0 : normal renal interstitium without inflammation infiltration; 1 : mild infiltration with little inflammatory cells scattered in renal interstitium; 2 : medium infiltration with large inflammatory cells cluster accumulated in renal interstitium; 3: severe infiltration with necrosis and fibrotic lesions.

\section{Immunohistochemical Staining}

Renal sections were dewaxed in xylene and dehydrated in alcohol. After antigen retrieval by microwaving in citric saline at $95^{\circ} \mathrm{C}$ for $3 \mathrm{~min}$, sections were treated with $3 \%$ hydrogen peroxide for $25 \mathrm{~min}$. The sections were blocked with $5 \%$ BSA and then incubated at $4{ }^{\circ} \mathrm{C}$ in a moist chamber overnight with primary antibody against mouse anti-Aminopeptidase P (JG-12) antibody (1:1000). Then sections were incubated with HRPconjugated goat anti-mouse IgG (DAKO, Wuhan, China) for $50 \mathrm{~min}$ at room temperature. JG-12 expressions were visualized by DAB (DAKO, Wuhan, China) staining. Sections were then examined under an optical microscope (Nikon ECLIPSE Ti-U, Nikon Corporation, Tokyo, Japan). Fifteen randomly selected glomeruli were digitally captured (400× magnification) and the optical density was quantified by Image J 4.5 image software (NIH, USA).

\section{Cell Culture}

Primary human renal glomerular endothelial cells (HRGECs) were purchased from ScienCell Research Laboratories (Carlsbad, CA, USA) and were cultured in endothelial cell medium supplemented with the endothelial cell growth factor containing $5 \%$ of fetal bovine serum (FBS) (Gibco, Australia) in humidified air at $37{ }^{\circ} \mathrm{C}$ with $5 \% \mathrm{CO}_{2}$.

\section{KARGER}




\section{Cellular Physiology Cell Physiol Biochem 2017;44:2378-2394 \begin{tabular}{l|l} 
and Biochemistry POI: 10.1159/000486154 & $\begin{array}{l}\text { (C) } 2017 \text { The Author(s). Published by S. Karger AG, Basel } \\
\text { www.karger.com/cpb }\end{array}$
\end{tabular} \\ Hou et al.: Renoprotective Effects of Salvianolic Acid A}

Trans-endothelial Protein Passage and Trans-endothelial Electrical Resistance Measurement

HRGECs were seeded on collagen-coated supports $\left(0.4 \mu \mathrm{m}\right.$ pore size, $0.33 \mathrm{~cm}^{2}$ surface area) in transwell inserts (Corning Incorporated Life Sciences, USA) and cultured until confluent, as previously reported [27]. After exposed to AGEs $(100 \mu \mathrm{g} / \mathrm{ml})$ or AGEs $(100 \mu \mathrm{g} / \mathrm{ml})$ with SalA $(0.3 \mu \mathrm{M}$ or $3 \mu \mathrm{M})$ for $24 \mathrm{~h}$, HRGECs trans-endothelial electrical resistance (TEER) was measured using Millipore Millicell ERS (Merck Millipore, USA) with probe MERSSTX01 and ERS-2 Epithelial volt-ohm meter as previously described [28]. Cell permeability was evaluated by passage of FITC-dextran (Sigma, USA). FITC-dextran $200 \mu \mathrm{l} \mathrm{of} 20 \mathrm{mg} / \mathrm{ml}$ in serum-free medium was added to the top chamber. One hour later, samples were removed from the top and bottom chamber and read in fluorometer (Molecular Devices Spectra Max M5, USA) at excitation 485 $\mathrm{nm}$ and emission $520 \mathrm{~nm}$.

\section{Cellular Reactive Oxygen Species Production Measurement}

To assess the production of reactive oxygen species (ROS), HRGECs were incubated with AGEs (100 $\mu \mathrm{g} / \mathrm{ml})$ or AGEs $(100 \mu \mathrm{g} / \mathrm{ml})$ with SalA $(0.3 \mu \mathrm{M}$ or $3 \mu \mathrm{M})$ for $3 \mathrm{~h}$, then cells $\left(1 \times 10^{6}\right)$ were stained with $10 \mu \mathrm{M}$ 2',7'-dichlorofluorescin diacetate (DCFDA) for $30 \mathrm{~min}$ at $37{ }^{\circ} \mathrm{C}$ and were washed and resuspended in $0.1 \mathrm{M}$ PBS, pH7.4. The oxidative conversion of DCFDA to DCFH-DA was measured by flow cytometry (C6, BD, USA).

\section{F-actin Immunofluorescence Staining}

HRGECs were treated with AGEs $(100 \mu \mathrm{g} / \mathrm{ml})$ or AGEs $(100 \mu \mathrm{g} / \mathrm{ml})$ with SalA $(0.3 \mu \mathrm{M}$ or $3 \mu \mathrm{M})$ for 24 h. F-actin distribution was assessed by staining with rhodamine-phalloidin 123 (RH123), followed by incubation with hoechst to stain the nuclear as previously described [29]. After staining, the cells were observed under a fluorescence microscope (Nikon ECLIPSE Ti-U, Nikon Corporation, Tokyo, Japan).

\section{RNA extraction and quantitative real-time PCR}

Total RNA was extracted using Trizol (Life Technologies, Grand Island, NY), followed by reverse transcription from $1 \mu \mathrm{g}$ of total RNA to cDNA. cDNA was synthesized using a high capacity cDNA reverse transcription kit (Applied Biosystems, Foster city, CA). cDNA subsequently underwent quantitative realtime polymerase chain reaction (PCR) using the CFX96TM Real-time System (Bio-Rad, Singapore). The sequences of the primers are as follows: ACCCAAGATGTGGCCTTTAG and GTGACAACAGCGAGGTGTAA for VE-cadherin, CAGCTGGTCTTTACTCCATCG and CAGATTCTTAGCCTTCCCACTC for claudin-5, TGATGCCTTATGGCACTGGACTCA and CTGCTGCCTTTGTTGCTCTTCCAA for TBP.

\section{Western Blot Analysis}

Total cell or rat kidney cortex tissue lysates $(20-50 \mu \mathrm{g})$ were subjected to SDS polyacrylamide gel. Electrophoresis and blotting were performed as described [30]. Signals were scanned and visualized by ChemiDoc-lTR 510 image system (Upland, CA, USA). The ratio of the protein interested was subjected to actin and was densitometrically analyzed by Image J software (NIH, MD).

\section{Statistical analysis}

All data are expressed as mean \pm SEM. One-way analysis of variance (ANOVA) with a post hoc Dunnett's test was used to determine statistically significant differences among groups. A value of $P<0.05$ was considered as statistically significant.

\section{Results}

\section{SalA inhibited AGEs formation and increased AGEs breaking}

Basing on the established AGEs screening model in our lab, the effect of SalA on the formation and breaking of AGEs were tested. Incubation with SalA effectively broke the formed AGEs dose-dependently (Fig. 2A) and exerted an inhibitory effect on AGEs formation (Fig. 2B).

SalA ameliorated metabolitic parameters in diabetic rats

Since AGEs contribute to diabetic nephropathy and SalA inhibits formation and breaking of AGEs, we next investigated the effects of SalA on diabetic nephropathy in vivo. Throughout 
eight-week administration, diabetic rats showed significant decrease in body weight (Fig. 3A) and increase in food and water intake (Fig. 3B, 3C). Treatment with SalA increased body weight by $11.7 \%$ compared with diabetic rats and reduced 24 -h food intake without statistical significance, and reduced $24 \mathrm{~h}$-water intake significantly. SalA decreased blood glucose from $4^{\text {th }}$ week till the end of experiment period (Fig. 3D), consistent with lowering serum GSP at $8^{\text {th }}$ week (Fig. 3E). Serum lipid profiles including TG, TCHO and LDL were increased in diabetic rats compared with normal rats. SalA treatment significantly decreased serum level

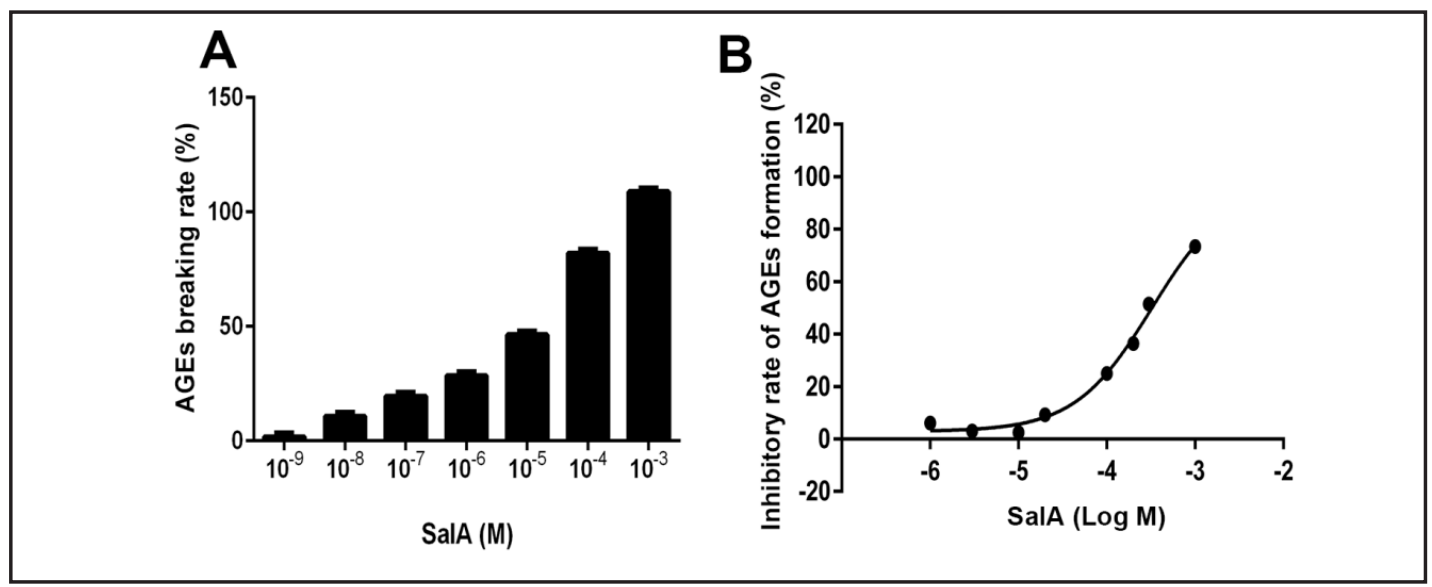

Fig. 2. SalA inhibited the formation and increased breaking of advanced glycation end products. (A) The breaking rate of SalA on formed AGEs. (B) The inhibitory rate of SalA on AGEs formation. Data are presented as mean $\pm \mathrm{SEM}$ of three independent experiments.

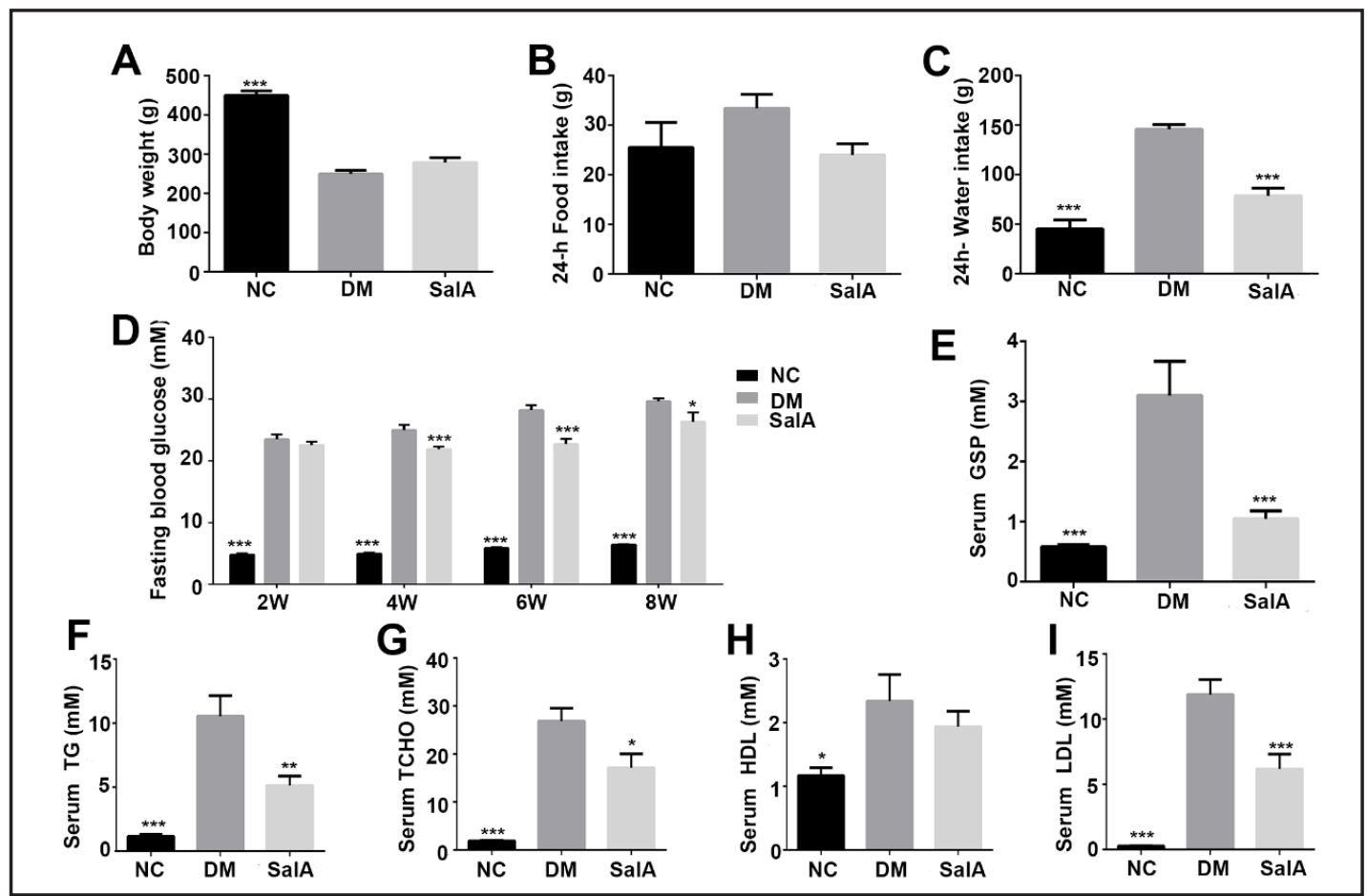

Fig. 3. SalA treatment ameliorated general parameters in diabetic rats. SalA $1 \mathrm{mg} / \mathrm{kg}$ was orally administered to diabetic rats for eight weeks and the general parameters of diabetic were assessed. (A) Body weight. (B) 24 h-food intake. (C) 24 h-water intake. (D) Fasting blood glucose. Serum level of GSP (E), TG (F), TCHO (G), HDL (H), and LDL (I) were measured. Data are presented as mean \pm SEM, $\mathrm{n}=8-10 .{ }^{*} P<0.05,{ }^{* *} P<0.01$, *** $P<0.001$ compared with DM group. 
of TG, TCHO and LDL (Fig. 3F-3I). Generally, SalA ameliorated the general diabetic conditions in type 2 diabetic rats.

\section{SalA protected against diabetic nephropathy in type 2 diabetic rats}

Compared with normal rats, diabetic rats showed significant increased kidney coefficient and 24 h-urine volume. SalA failed to impact kidney coefficient but markedly reduced 24 h-urine volume (Fig. 4A-4C). In addition, SalA dramatically ameliorated the glomerular hyperfiltration and renal function, shown by reduction of 24 h-urinary microalbumin, serum BUN and Scr (Fig. 4D-4F). Consistently, SalA administration increased creatinine clearance and blunted UACR elevation with statistically significance (Fig. 4G, 4H).

In accordance with urinalysis and blood analysis, SalA treatment ameliorated the pathological deteriorations of glomerulus as implied by histological examination PAS staining. The results showed obvious glomerular hypertrophy with significant increase in glomerular

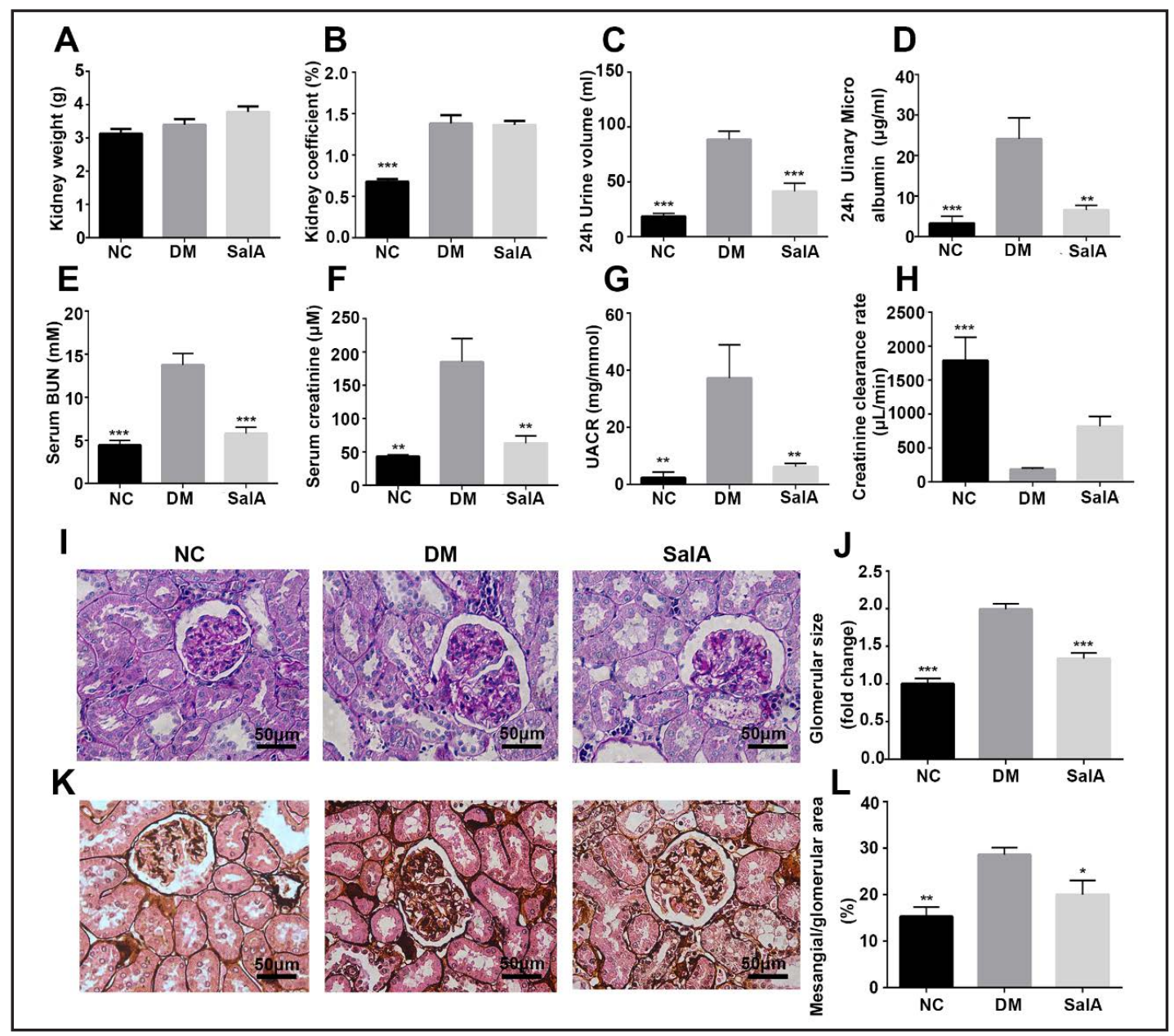

Fig. 4. SalA treatment alleviated diabetic glomerular hyperfiltration and kidney structural deterioration. SalA $1 \mathrm{mg} / \mathrm{kg}$ was orally administered to diabetic rats for eight weeks. Kidney weight (A), Kidney coefficient (B), 24-h urine volume (C), 24-h urinary albumin (D), Serum level of BUN (E), and creatinine (F), Urine $\mathrm{ACR}(\mathrm{G})$, and Creatinine clearance rate $(\mathrm{H})$ were determined. Data are presented as mean $\pm \mathrm{SEM}, \mathrm{n}=8$ 10. ${ }^{* *} P<0.01,{ }^{* * *} P<0.001$, compared with DM group. (I) Representative photomicrographs of PAS stained kidneys, magnified by 400×. (J) Quantitative analysis of glomerular size in three groups. (K) Representative photomicrographs of PASM-stained kidneys, magnified by 400×. (L) Quantitative analysis of mesangial matrix expansion in three groups. 15 glomeruli were calculated in each slide, data are presented as mean \pm SEM, $\mathrm{n}=4 .{ }^{*} P<0.05,{ }^{* *} P<0.01,{ }^{* * *} P<0.001$, compared with DM group. 


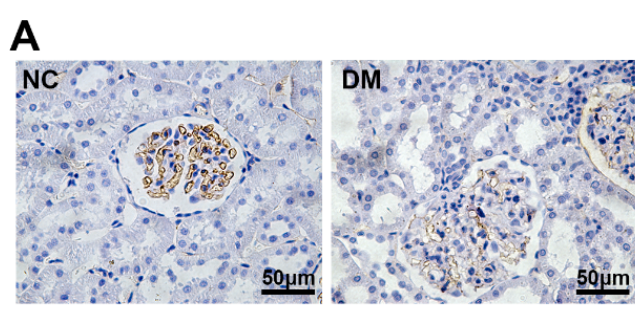

C

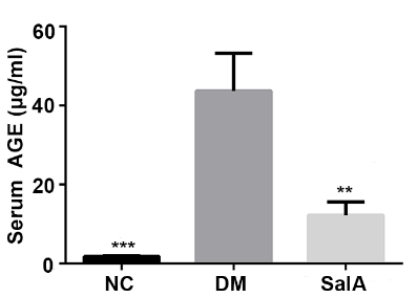

$\mathbf{F}$

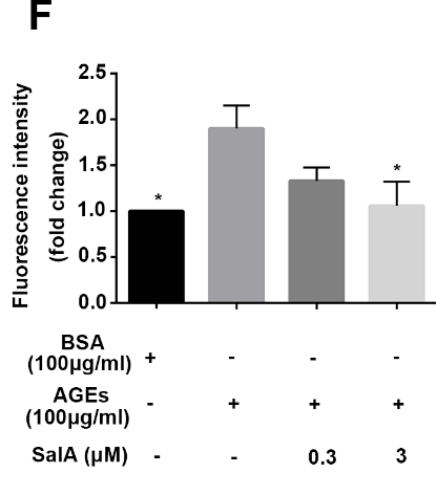

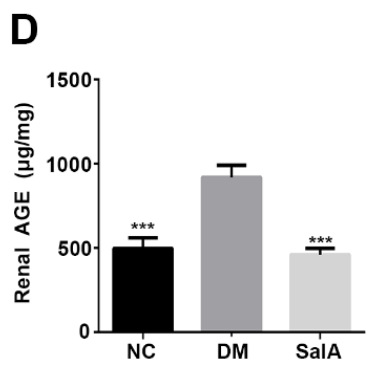

\section{G \\ G}

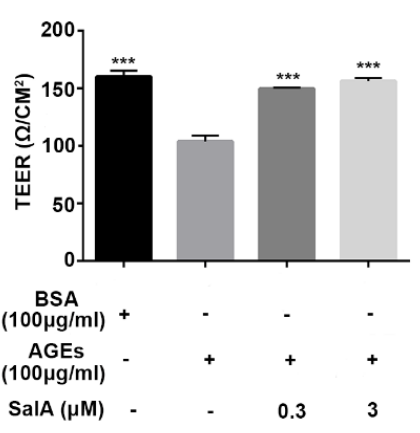

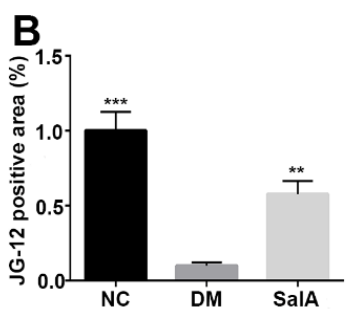

E

RAGE

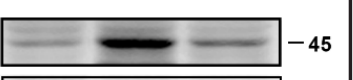

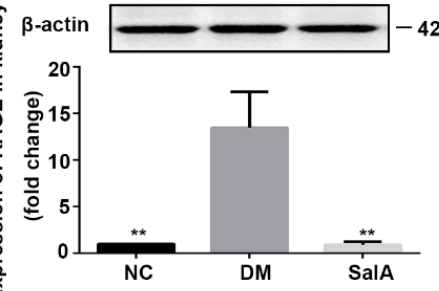

H
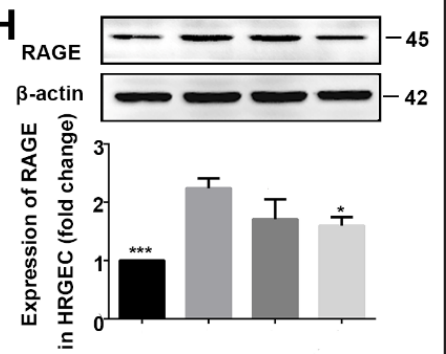

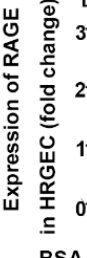

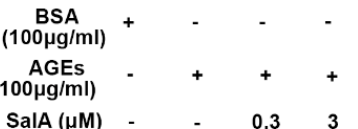

Fig. 5. SalA protected glomerular endothelium through inhibiting AGE-RAGE. (A) Immunohistochemical staining of JG-12, magnified by $400 \times$. (B) Quantitative analysis of JG-12 positive cells. 15 glomeruli were calculated in each slide, data are presented as mean $\pm \mathrm{SEM}, \mathrm{n}=4$. ${ }^{* *} P<0.01,{ }^{* * *} P<0.001$, compared with DM group. The concentration of AGEs in serum (C) and in renal cortex (D) were measured. (E) Western blot analysis for RAGE expression in renal cortex. Data are presented as mean \pm SEM, $n=6-8$ per group. ${ }^{* *} P<0.01,{ }^{* * *} P<0.001$, compared with DM group. HRGECs were incubated with AGEs $(100 \mu \mathrm{g} / \mathrm{ml})$ or AGEs $(100 \mu \mathrm{g} / \mathrm{ml})$ with SalA $(0.3 \mu \mathrm{M}$ or $3 \mu \mathrm{M})$ for $24 \mathrm{~h}$, transwell assay and TEER measurement were conducted as described in method. (F) The permeability of HRGECs monolayer for FITC-dextran determined by transwell assay. Data showed FITC-dextran fluorescence intensity ratio fold change of lower chamber to up chamber. (G) The electrical resistance of HRGECs monolayer assessed by Millicell-ERS ohmmeter. (H) Western blot analysis for RAGE expression in HRGECs exposed in AGEs $(100 \mu \mathrm{g} / \mathrm{ml})$ or AGEs $(100 \mu \mathrm{g} / \mathrm{ml})$ with SalA $(0.3 \mu \mathrm{M}$ or $3 \mu \mathrm{M})$ for $24 \mathrm{~h}$. Data are presented as mean \pm SEM of three independent experiments. ${ }^{*} P<0.05$, *** $P<0.001$, compared with AGEs group.

size of diabetic rats. SalA treatment effectively prevented these histological changes (Fig. 4I, 4J). The mesangial matrix accumulation was more extensive in the glomerulus of diabetic rats than normal rats exhibited by PASM staining, and 8-week treatment of SalA significantly reduced such expansion (Fig. 4K, 4L).

SalA protected glomerular endothelium function through inhibiting AGE-RAGE signaling

Changes in endothelium expression patterns of experimental rats' glomerulus were examined by immunohistochemical staining of tissue sections with JG-12, a murine IgG1 monoclonal antibody specific for rat aminopeptidase P. As shown in JG-12 staining (Fig. 5A, 
5B), we observed significant reduction of glomerular endothelium in diabetic kidney, while SalA hindered this loss and almost restored the endothelium to normal level. To investigate whether the glomerular protection was related to inhibiting AGE-RAGE, we determined the AGEs level and RAGE expression. The results suggested that SalA significantly reduced both serum and renal cortex AGEs accumulation and downregulated RAGE expression in kidney as well (Fig. 5C-5E).

In accordance with in vivo results, SalA alleviated higher permeability to FITC-dextran in AGEs-exposed monolayer of glomerular endothelial cells, as evidenced by lower ratio of fluorescence extracted from down chamber to up chamber in transwell assay (Fig. 5F). In addition, decreased TEER value suggested that an impaired extracellular electronic barrier induced by AGEs was also ameliorated by SalA $0.3 \mu \mathrm{M}$ and $3 \mu \mathrm{M}$ (Fig. 5G). Consistently, SalA downregulated RAGE expression in AGEs-injured HRGECs (Fig. 5H). Collectively, these results indicated that SalA exerted endothelial protection through its inhibition of AGE-RAGE.

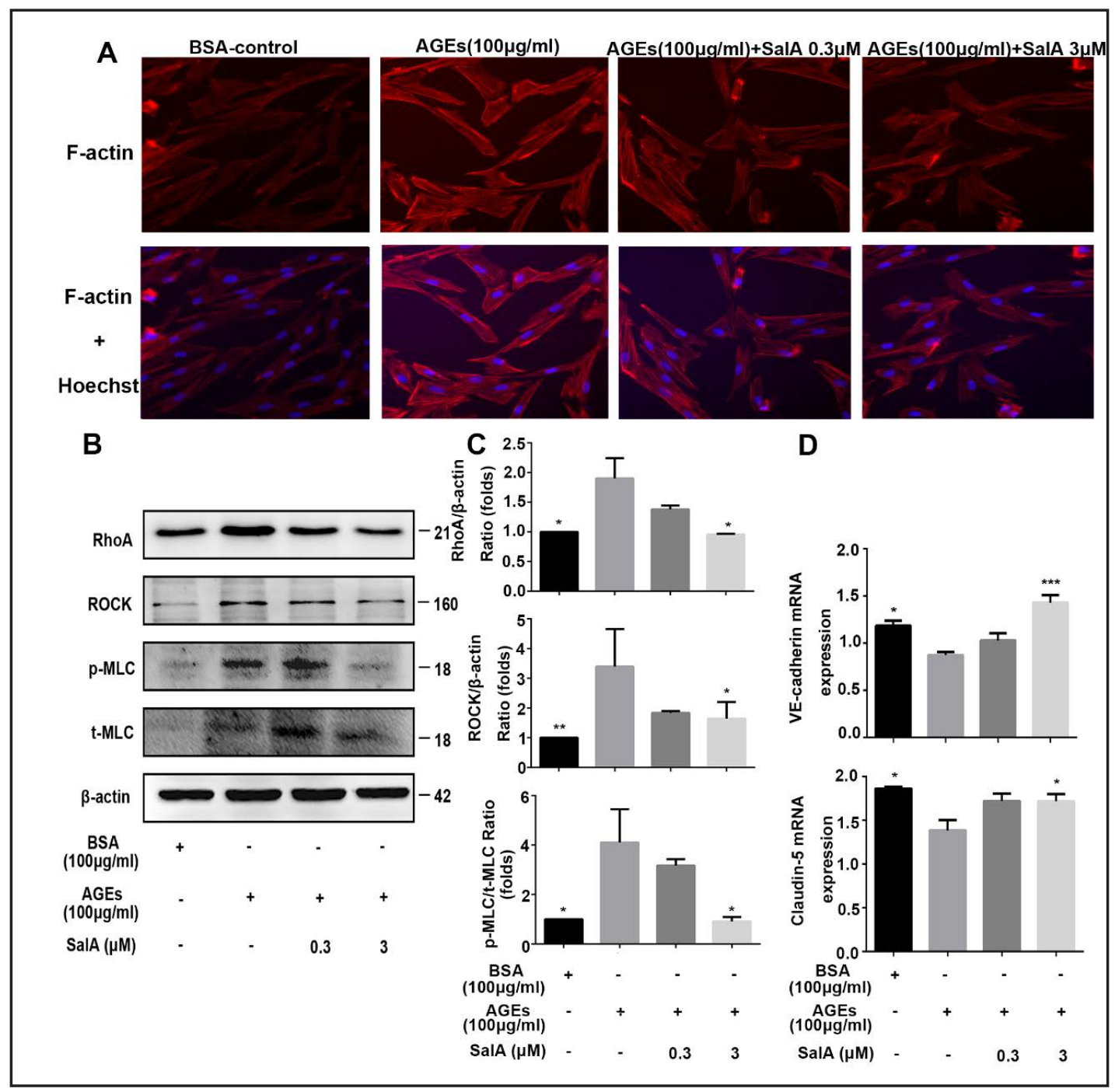

Fig. 6. SalA hindered rearrangement of actin cytoskeleton through inhibiting AGEs induced activation of RhoA-ROCK pathway. HRGECs were incubated with AGEs $(100 \mu \mathrm{g} / \mathrm{ml})$ or AGEs $(100 \mu \mathrm{g} / \mathrm{ml})$ with SalA $(0.3$ $\mu \mathrm{M}$ or $3 \mu \mathrm{M}$ ) for $24 \mathrm{~h}$. (A) Fluorescence staining of F-actin was conducted as described in method, (B) \& (C) Western blot analysis for RhoA, ROCK, p-MLC and t-MLC expression in HRGECs after treatment for $24 \mathrm{~h}$. (D) qPCR results of VE-cadherin and claudin- 5 in HRGECs after treatment for $24 \mathrm{~h}$. Data are presented as mean \pm SEM of three independent experiments. ${ }^{*} P<0.05,{ }^{* *} P<0.01$, compared with AGEs group.

\section{KARGER}




\section{Cellular Physiology Cell Physiol Biochem 2017;44:2378-2394

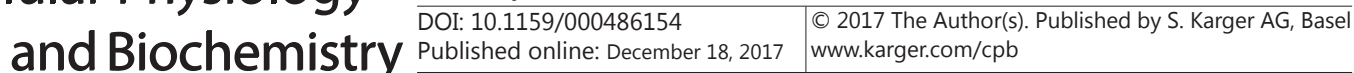 \\ Hou et al.: Renoprotective Effects of Salvianolic Acid A}

SalA restored glomerular endothelial permeability through suppressing AGEs-induced rearrangement of actin cytoskeleton via AGE-RAGE-RhoA/ROCK pathway

We next focused on the endothelial morphological changes in cytoskeleton to elucidate the protective effects of SalA on restoration of glomerular endothelial permeability. F-actin staining of HRGECs revealed that, BSA control group showed less fiber string inside the cell with a slight ring around the cell periphery, however after exposed to AGEs $100 \mu \mathrm{g} /$ $\mathrm{ml}$, the fiber string was stronger and became fascicle. Treatment with SalA $0.3 \mu \mathrm{M}$ and $3 \mu \mathrm{M}$ alleviated this alteration (Fig. 6A).

Activation of RhoA-ROCK is the main mechanism involved in the AGEs-induced disarrangement of F-actin, so we examined the RhoA pathway by western blot. It suggested that RhoA and ROCK expression were upregulated after AGEs injury. Treatment with SalA 3 $\mu \mathrm{M}$ significantly suppressed the activated RhoA and ROCK expression. MLC is the downstream pathway of RhoA-ROCK and involved in rearranging cytoskeleton. The phosphorylation of MLC was also reduced by SalA $3 \mu \mathrm{M}$ (Fig. 6B, 6C). We next examined the expression of VEcadherin and claudin-5 in HRGECs, which is downregulated by RhoA-ROCK-MLC pathway, and are of vital importance in maintaining the normal permeability of endothelial cell. The results showed that in AGEs-exposed HRGECs, VE-cadherin and claudin-5 were markedly decreased while treatment with SalA could restore the cytoskeleton protein (Fig. 6D). Collectively, SalA restored glomerular endothelial permeability through suppressing rearrangement of actin cytoskeleton via AGE-RAGE-RhoA/ROCK pathway.

SalA attenuated AGEs-induced oxidative stress in glomerular endothelial cell and diabetic rats via AGE-RAGE-Nox4 signaling

AGEs-induced ROS production is the main resource of oxidative stress, which we examined using DCFDA by flow cytometry. As displayed in Fig. 7A and 7B, AGEs significantly elevated ROS, while both SalA $0.3 \mu \mathrm{M}$ and $3 \mu \mathrm{M}$ scavenged ROS production induced by AGEs. NADPH oxidase played a central role in AGEs-injured HRGECs [9], we found that Nox4 was highly expressed and SalA downregulated Nox4 expression (Fig. 7C).

In accordance with in vitro results, SalA downregulated the Nox4 expression in diabetic kidney (Fig. 7D). Moreover, SalA restored the reduced enzyme activity of CAT in diabetic kidney (Fig. 7E). The effect of SalA on attenuating oxidative stress in diabetic rats was assessed by measuring serum levels of MDA, 8-OHdG and activity of SOD. As shown in Fig. 7F-7I, oxidative stress in diabetic model group is much more severe than normal group, administration of SalA restored the oxidative stress by lowering the level of MDA, 8-OHdG and NO, as well as enhanced serum SOD. In sum, SalA attenuated AGEs-induced oxidative stress in glomerular endothelial cell and diabetic rats via AGE-RAGE-Nox4 signaling.

SalA alleviated inflammation and restored the disturbed autophagy in diabetic rats

Since we observed a marked protection against AGEs-induced ROS production, we set out to assess whether SalA has effect on inflammation and autophagy in diabetic rats, as downstream of oxidative stress. The inflammation infiltration in kidney estimated by HE staining showed that diffused inflammatory cells clustered in interstitial tissue of kidney, SalA mildly reduced this inflammatory infiltration (Fig. 8A). We then examined the protein expression of proinflammatory cytokines including TNF- $\alpha$, IL-6, IL-1 $\beta$, ICAM-1, and VCAM- 1 in the kidney. The proinflammatory cytokines expression was significantly upregulated in diabetic kidneys, while SalA downregulated the proinflammatory cytokines compared with the diabetic model group (Fig. 8B).

The release of proinflammatory cytokines combined with environment stress such as oxidative stress undergoes integration to ensure tissue homeostasis. Autophagy is a major contributor to maintain intracellular homeostasis. p62/Sqstm1, which is accumulated by disturbed autophagy lysosome pathway, was significantly increased in the kidneys of diabetic rat. Meanwhile, the ratio of LC3II to LC3I is lower accompanied with decreased Bclin-1. These results suggested that autophagy lysosome pathway was impaired during diabetic nephropathy progress. However, this impairment was restored by eight-week administration 


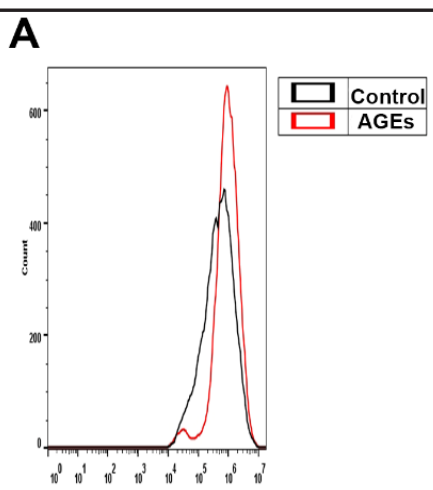

C
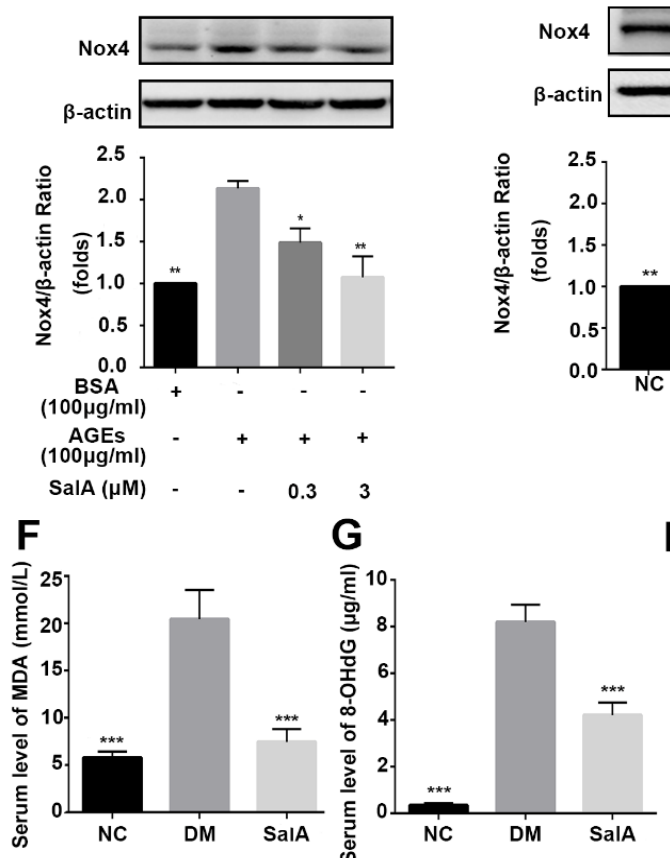

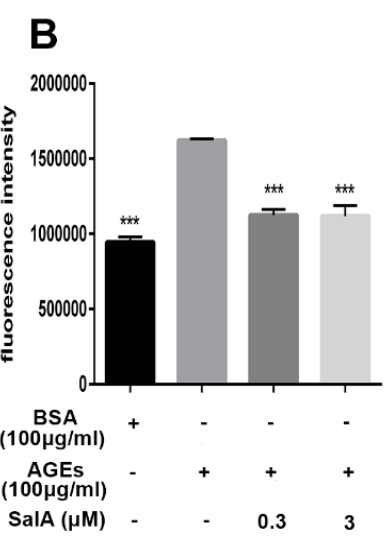

E
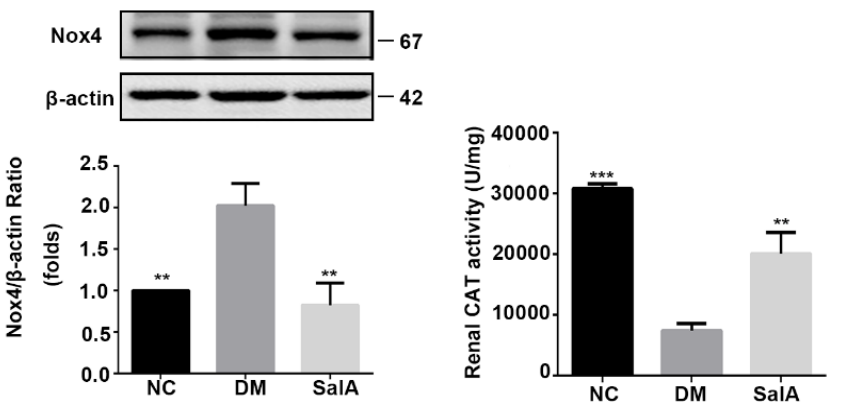

Fig. 7. SalA attenuated AGEs-induced oxidative stress in glomerular endothelial cell and diabetic rats via AGE-RAGE-Nox4 signaling. (A, B) ROS production in HRGECs after exposed in AGEs $(100 \mu \mathrm{g} / \mathrm{ml})$ or AGEs $(100 \mu \mathrm{g} / \mathrm{ml})$ with SalA $(0.3 \mu \mathrm{M}$ or $3 \mu \mathrm{M})$ for $3 \mathrm{~h}$ and was analyzed by flow cytometry. (C) Western blot analysis for Nox4 expression in HRGECs after treatment for $24 \mathrm{~h} . \mathrm{n}=3$ per group, replicated twice. Data are presented as mean \pm SEM of three independent experiments. ${ }^{*} P<0.05,{ }^{* *} P<0.01,{ }^{* * *} P<0.001$, compared with AGEs group. (D) Western blot analysis for Nox4 expression in renal cortex of diabetic rats. Data are presented as mean + SEM, $n=6$ per group, replicated twice. Activity of renal CAT (E), serum MDA (F), serum 8-OHdG $(\mathrm{G})$, serum NO $(\mathrm{H})$ and serum SOD $(\mathrm{I})$ in diabetic rats were determined. Data are presented as mean $\pm \mathrm{SEM}, \mathrm{n}=8 .{ }^{* *} P<0.01,{ }^{* * *} P<0.001$, compared with the DM group.

of SalA (Fig. 8C). Previous studies have confirmed that Sirt1 exerts renal protective effects involved in regulating cellular homeostasis such as autophagy and inflammation. In our current study, Sirt1 expression was dramatically downregulated in diabetic nephropathy, and correspondingly a key mediator in Sirt1-Foxo3a-Bnip3 related regulation of autophagy. Treatment with SalA restored Sirt1 expression and upregulated its downstream mediator expression, and subsequently upregulated Atg5, Atg7, and Atg12 which were important in autophagosome formation (Fig. 8D). Collectively, our findings indicated that SalA upregulated Sirt1-Foxo3a-Bnip3 pathway to ameliorate the impaired autophagy. 


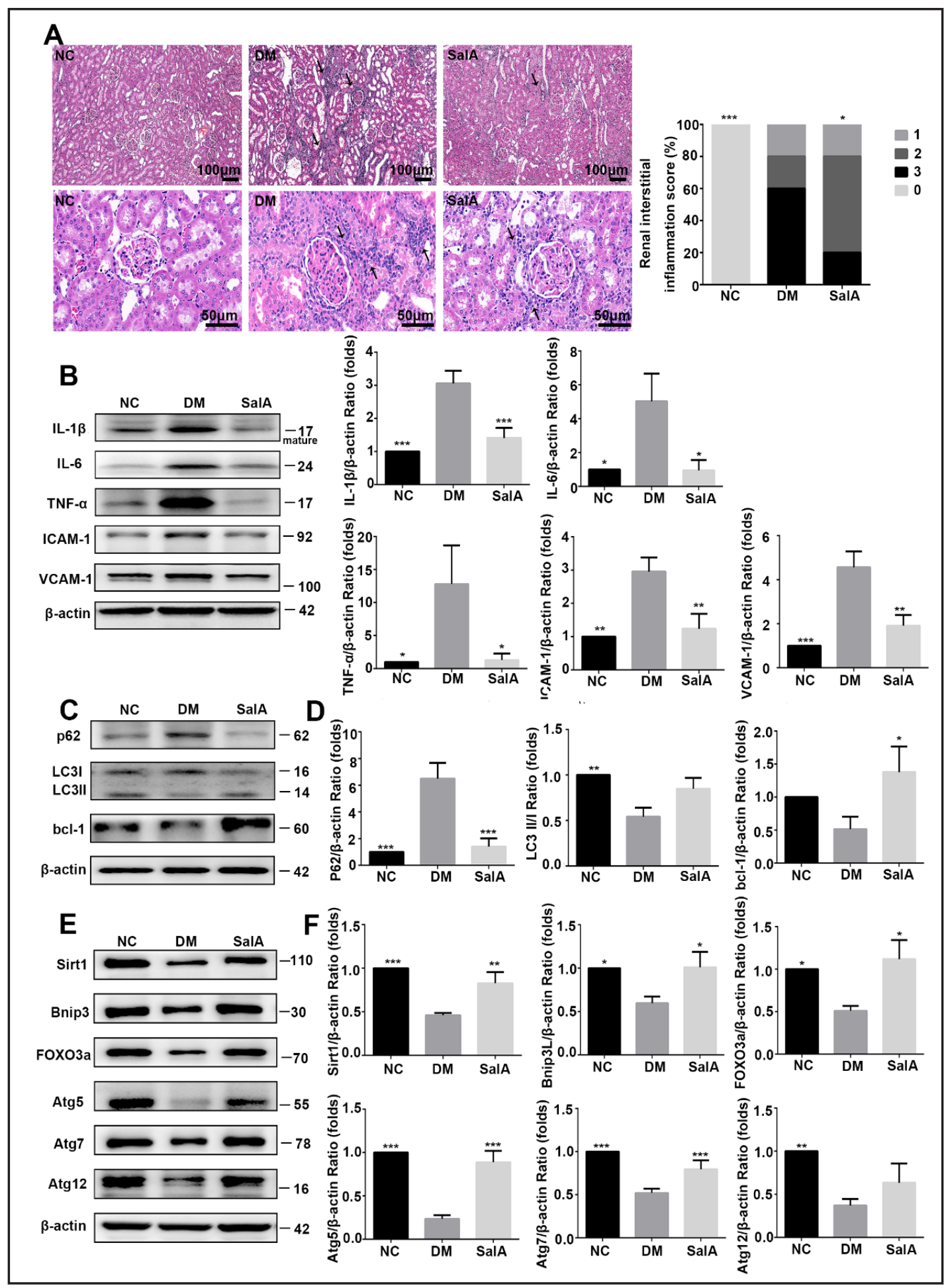

Fig. 8. SalA reduced renal inflammation and ameliorated Sirt1-related autophagy in diabetic kidney. (A) Representative pathological changes of renal inflammation were assessed by HE staining. (100×, 400×), and the inflammation score quantified as mentioned in method, $n=4$. (B) Western blot analysis for TNF- $\alpha$, IL-6, IL-1 $\beta$, ICAM-1, and VCAM-1 expression in renal cortex of diabetic rats. Data are presented as mean \pm SEM, $\mathrm{n}=6$ per group, replicated twice. The expression and quantification of autophagy marker (C, D) and Sirt1related regulator of autophagy (E,F) in kidney was analyzed by western blot. Data is presented as mean \pm SEM. $\mathrm{n}=6$ per group, replicated twice. ${ }^{*} P<0.05,{ }^{* *} P<0.01$, ${ }^{* * *} P<0.001$, compared with the DM group.

\section{KARGER}




\section{Discussion}

It is well known that AGEs and its receptor RAGE play important roles in vascular endothelial dysfunction, diabetic complications, especially in diabetic nephropathy. The endothelial protection of SalA proved previously [16] led us to detect its effects on glomerular endothelium and investigate its influence on AGE-RAGE signaling. Based on the previous findings of SalA on type 2 diabetic complications in our lab, we chose SalA $1 \mathrm{mg} / \mathrm{kg} /$ day as the treatment dose in our current study. The present study first reported that SalA inhibited AGEs formation, and AGE-RAGE signaling as well. We demonstrated that SalA protected against early-stage diabetic nephropathy by attenuating glomerular hyperfiltration and kidney structure deterioration. Further mechanism investigation suggested that the protective effects of SalA on glomerular microvascular endothelial cell were involved in suppressing AGEs-activated RhoA-ROCK and subsequent phosphorylation of MLC-regulated disarrangement of cytoskeleton. More importantly, these beneficial effects of SalA were involved in attenuating AGE-RAGE-Nox4-induced oxidative stress, inflammation and enhancing autophagy of glomerular endothelial cell in type 2 diabetic rats.

Among all impact factors contributing to DN, AGEs-induced structural and functional damage in kidney is hyperglycemia-independent [31]. Thus, intensive blood-glucose control could barely prevent the progress of diabetic nephropathy [32]. Various types of AGEs formation inhibitors (e.g. aminoguanidine) or AGEs breakers (e.g. alagebrium) have shown the beneficial effects on diabetic nephropathy through decreasing collagen accumulation and subsequently inhibiting glomerulosclerosis injury to retard albumin excretion rate [33]. However, these compounds have been withdrawn from clinical trial because of its unsatisfactory safety [34]. In current study, we used SalA, a natural product from Salvia miltiorrhiza Bunge with the confirmed drug safety. The screening results showed the direct inhibitory effect of SalA on AGEs. Different from aminoguanidine, a nonspecific inhibitor via binding early glycation and glycoxidation products, SalA not only inhibited AGEs formation but also broke the formed AGEs dose-dependently. Our study confirmed that SalA suppressed AGEs accumulation in both serum and kidney tissue. Moreover, SalA reduced RAGE expression in AGEs-injured glomerular endothelial cells and kidney in diabetic rats, which is possibly owing to SalA's inhibitory effect on AGEs since AGEs can induce RAGE expression dose-dependently and time-dependently [35].

Current therapeutic strategies for diabetic nephropathy are trying to slow the disease progression although unable to reverse the disease. In the present eight-week study, we observed the obvious glomerular hyperfiltration and albuminuria in diabetic rats, accompanied by markedly accentuated glomerular hypertrophy and mesangial matrix expansion, which is characteristic of early-stage diabetic nephropathy. Eight-week treatment with SalA effectively ameliorated the glomerular hyperfiltration and urinary microalbumin as well as suppressed the glomerular hypertrophy and mesangial matrix expansion, which suggested that an early therapeutic intervene might slow down the progression of diabetic nephropathy.

In Maricbilkan's research [36], they found that initiating events of renal injury largely related with the damage, loss, and remodeling of microvascular in the diabetic kidney. We confirmed this significant loss of glomerular microvascular endothelium in diabetic rats evidenced by low JG-12 expression. SalA restored the endothelium loss toward normal level. AGEs-provoked disruption of tight junctions and disarrangement of the F-actin cytoskeleton contribute to endothelial hyperpermeability. In this study, the hyperpermeability and impaired extra cellular barrier induced by AGEs were ameliorated by SalA. RhoA/ROCKmediated disarrangement of actin cytoskeleton and alteration of cell contractility are involved in endothelial hyperpermeability in response to AGEs injury [29]. Activation of RhoA-ROCKMLC will downregulate VE-cadherin and claudin-5, which is central in permeability changes in chiefly organizing endothelial barrier [37]. Previous studies demonstrated that activation of RhoA depends on the binding of AGEs to RAGE [38]. In this study, we observed F-actin disarrangement in AGEs-injured HRGECs and SalA downregulated the AGEs-activated 
RhoA-ROCK, phosphorylation of MLC and subsequently restored VE-cadherin and claudin-5 expression, which may be the underlying mechanism that SalA restored the endothelial permeability subjected to AGEs injury.

Oxidative stress is one of major pathogenesis contributing to diabetic complications including nephropathy. It has been proved that increased oxidative stress induced glomerular endothelial surface layer deterioration, resulting in exacerbation of glomerular permselectivity and development of albuminuria in Zucker fatty rat models [39]. NADPH oxidases play important role in glucose-induced generation of ROS [40], however, AGEs increase the levels of ROS through activation of NADPH oxidase in a receptor-independent manner or through RAGE. Nox4, which is the most abundant NADPH oxidase homolog in kidney, is a major source of renal ROS [41]. Previous findings showed that SalA has a profound antioxidant effect on scavenging free radical [42] and activating antioxidase regulator such as Nrf2 [26]. In accordance with our previous study, we demonstrated SalA downregulated Nox4 expression aroused by AGEs and scavenged ROS production markedly in glomerular endothelial cell. Taken together, the protection of SalA on early-stage diabetic nephropathy may be largely due to its antioxidative effect by AGE-RAGE-Nox4 signaling pathway.

ROS is the most important mechanism for inflammatory response induction. Moreover, inflammation is also a vital mechanism leading to endothelial injury in diabetic context since it is often strongly linked to intensify oxidative stress-induced endothelial damage [22]. In this study, we found that SalA markedly reduced the production of proinflammatory cytokines such as IL- 6 and TNF- $\alpha$ and inflammatory infiltration in diabetic kidney. It is possibly because SalA inhibited AGEs induced-ROS cascades and subsequently attenuated the inflammation. However, this hypothesis needs a further demonstration in our future work.

In response to inflammation and oxidative stress, autophagy is of vital importance in maintaining cellular homeostasis [43]. It has been reported that autophagy is impaired in diabetic nephropathy [44] and has become a promising target for treatment [45]. We reported that a disturbed autophagy-lysosome pathway is related with AGEs-evoked oxidative stress which triggered lysosomal membrane permeabilization and lysosomal dysfunction [46]. Very likely, we observed a decline of autophagy in diabetic kidney indicated by accumulation of p62/Sqstm1, decline of the ratio of LC3II/LC3I, and Beclin-1. SalA mildly restored diabetic autophagy after eight-week administration. Considering the interplay among inflammation, oxidative stress and autophagy we discussed above in diabetic nephropathy, this amelioration of SalA might be closely related to its attenuation of oxidative stress and inflammation response. Previous research has confirmed that Sirt1-facilitated autophagy induction in diabetic nephropathy might contribute to a renoprotective effect [47]. Sirt1 expression is downregulated in diabetic patient [48]. In addition, the expression and activity of Sirt 1 is decreased in a dose- and time-dependent manner under AGEs injury [49]. In accordance with previous studies, we observed the downregulated Sirt1 expression in diabetic kidneys. SalA restored the Sirt1 expression and subsequently upregulated the expression of downstream Sirt1-Foxo3a-Bnip3 and key protein in autophagosome formation such as atg5, atg7 and atg12. Collectively, SalA inhibited AGEs induced-ROS cascades which contribute to a subsequently beneficial effect on attenuating the inflammation and restoring the disturbed autophagy.

\section{Conclusion}

Our current findings showed that SalA attenuated glomerular filtration dysfunction and protected against diabetic nephropathy by inhibiting AGE-RAGE signaling. Further investigation suggested that its protective effects on glomerular endothelial cell are involved in preventing AGEs-induced endothelial loss and stabilizing permeability as well as cell electrical barrier through suppressing AGE-RAGE-RhoA/ROCK mediated cytoskeleton rearrangement. Additionally, SalA exerted antioxidative effect, thereby ameliorated the inflammation and enhanced impaired autophagy by AGE-RAGE-Nox4 signaling pathway. In 


\section{Cellular Physiology Cell Physiol Biochem 2017;44:2378-2394 \begin{tabular}{l|l|l|}
\hline DOI: 10.1159/000486154 & $\begin{array}{l}\text { C) } 2017 \text { The Author(s). Published by S. Karger AG, Basel } \\
\text { www.karger.com/cpb }\end{array}$
\end{tabular}

Fig. 9. Schematic diagram showing that SalA ameliorated diabetic nephropathy through inhibiting AGE-RAGE system. AGEs interact with RAGE induces activation of RhoA/ROCK pathway, which results in cytoskeleton rearrangement and endothelial hyperpermeabilty. On the other hand, SalA attenuated oxidative stress through inhibition on AGE-RAGE-Nox4, which will subsequently suppress inflammation and an impaired autophagy in diabetic rats.

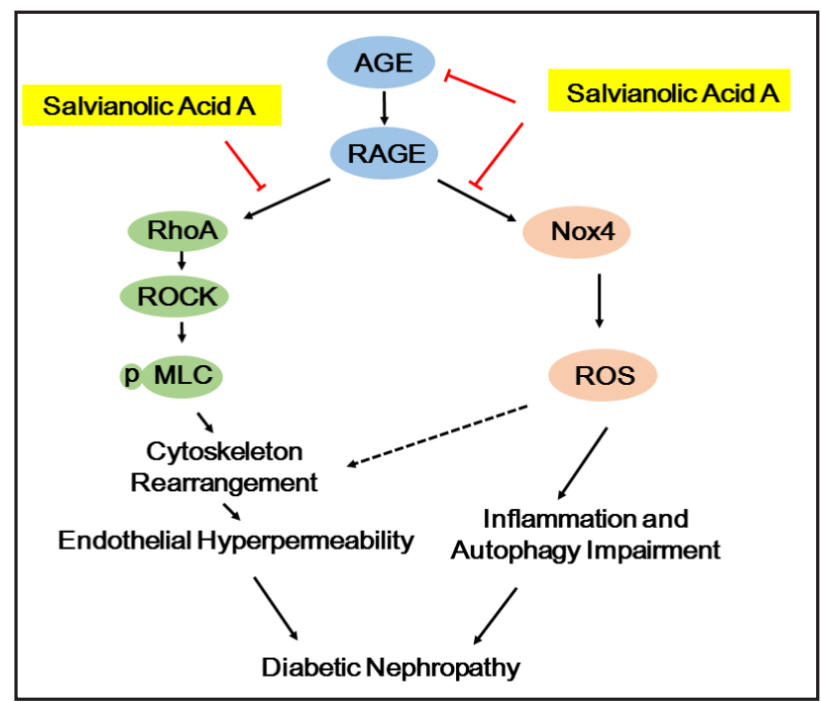

summary, compared with other AGEs formation inhibitors or breakers, SalA is a promising therapy for diabetic nephropathy with striking efficacy and safety, but still need further investigation (Fig. 9).

\section{Disclosure Statement}

The authors have no conflict of interest at all.

\section{Acknowledgements}

This project was funded by National Key Research and Development Plan (2016YFC1000905); CAMS Innovation Fund for Medical Sciences (CIFMS) (2016-I2M-3007, 2016-I2M-1-010); National Nature Science Foundation of China (81770847).

\section{References}

1 Hostetter TH: Hyperfiltration and glomerulosclerosis. Semin Nephrol 2003;23:194-199.

2 Soldatos G, Cooper ME: Diabetic nephropathy: important pathophysiologic mechanisms. Diabetes Res Clin Pract 2008;82 Suppl 1:S75-S79.

3 Satchell SC: The glomerular endothelium emerges as a key player in diabetic nephropathy. Kidney Int 2012;82:949-951.

4 Salmon AH, Satchell SC: Endothelial glycocalyx dysfunction in disease: albuminuria and increased microvascular permeability. J Pathol 2012;226:562-574.

5 Salameh A, Zinn M, Dhein S: High D-glucose induces alterations of endothelial cell structure in a cell-culture model. J Cardiovasc Pharmacol 1997;30:182-190.

6 Popov D: Endothelial cell dysfunction in hyperglycemia: Phenotypic change, intracellular signaling modification, ultrastructural alteration, and potential clinical outcomes. Int J Diabetes Mellit 2010;2:189195.

7 Thieme K, Da SK, Fabre NT, Catanozi S, Monteiro MB, Santosbezerra DP, Costapessoa JM, Oliveirasouza M, Machado UF, Passarelli M, Correa-Giannella ML: N-acetyl cysteine attenuated the deleterious effects of advanced glycation end-products on the kidney of non-diabetic rats. Cell Physiol Biochem 2016;40:608620.

8 Hirose A, Tanikawa T, Mori H, Okada Y, Tanaka Y: Advanced glycation end products increase endothelial permeability through the RAGE/Rho signaling pathway. FEBS Lett 2010;584:61-66.

-9 Wautier MP, Chappey O, Corda S, Stern DM, Schmidt AM, Wautier JL: Activation of NADPH oxidase by AGE links oxidant stress to altered gene expression via RAGE. Am J Physiol-Endocrinol Metab 2001;280:E685-E694. 


\section{Cellular Physiology Cell Physiol Biochem 2017;44:2378-2394 \begin{tabular}{l|l|l}
\hline and BOI: 10.1159/000486154 & $\begin{array}{l}\text { C } 2017 \text { The Author(s). Published by S. Karger AG, Basel } \\
\text { www.karger.com/cpb }\end{array}$ \\
\hline
\end{tabular}}

Hou et al.: Renoprotective Effects of Salvianolic Acid A

10 Huang CY, Lai KY, Hung LF, Wu WL, Liu FC, Ho LJ: Advanced glycation end products cause collagen II reduction by activating Janus kinase/signal transducer and activator of transcription 3 pathway in porcine chondrocytes. Rheumatology 2011;50:1379-1389.

11 Lin TJ, Zhang KJ, Liu GT: Effects of salvianolic acid A on oxygen radicals released by rat neutrophils and on neutrophil function. Biochem Pharmacol 1996;51:1237-1241.

12 Wang SB, Pang XB, Zhao Y, Wang YH, Zhang L, Yang XY, Fang LH, Du GH: Protection of salvianolic acid A on rat brain from ischemic damage via soluble epoxide hydrolase inhibition. J Asian Nat Prod Res 2012;14:1084-1092.

-13 Oh KS, Oh BK, Mun J, Seo HW, Lee BH: Salvianolic acid A suppress lipopolysaccharide-induced NF- $\kappa B$ signaling pathway by targeting IKK $\beta$. Int Immunopharmacol 2011;11:1901-1906.

14 Qiang GF, Yang XY, Shi LL, Zhang HA, Chen BN, Zhao Y, Zu M, Zhou D, Guo J, Yang HG, Zhang L, Du GH: Antidiabetic effect of salvianolic acid A on diabetic animal models via ampk activation and mitochondrial regulation. Cell Physiol Biochem 2015;36:395-408.

15 Qiang GF, Yang XY, Xuan Q Shi LL, Zhang HA, Chen BN, Li X, Zu M, Zhou D, Guo J, Yang HG, Zhang L, Du GH: Salvianolic acid A prevents the pathological progression of hepatic fibrosis in high-fat diet-fed and streptozotocin-induced diabetic rats. Am J Chin Med 2014;42:1183-1198.

16 Yang XY, Qiang GF, Zhang L, Zhu XM, Wang SB, Sun L, Yang HG, Du GH: Salvianolic acid A protects against vascular endothelial dysfunction in high-fat diet fed and streptozotocin-induced diabetic rats. J Asian Nat Prod Res 2011;13:884-894.

17 Yu XY, Zhang L, Yang XY, Huang H, Huang ZL, Shi LL, Zhang HA, Du GH: Salvianolic acid A protects the peripheral nerve function in diabetic rats through regulation of the AMPK-PGC1 $\alpha$-Sirt3 axis. Molecules 2012;17:11216-11228.

18 Fan HY, Yang MY, Qi D, Zhang ZK, Zhu L, Shangguan XX, Liu K, Xu H, Che X: Salvianolic acid A as a multifunctional agent ameliorates doxorubicin-induced nephropathy in rats. Sci Rep 2015;5.

19 Kishore L, Kaur N, Singh R: Renoprotective effect of Bacopa monnieri via inhibition of advanced glycation end products and oxidative stress in STZ-nicotinamide-induced diabetic nephropathy. Ren Fail 2016;38:1528-1544.

20 Tang LQ, Ni WJ, Cai M, Ding HH, Liu S, Zhang ST: Renoprotective effects of berberine and its potential effect on the expression of $\beta$-arrestins and intercellular adhesion molecule- 1 and vascular cell adhesion molecule-1 in streptozocin-diabetic nephropathy rats. J Diabetes 2016;8:693-700.

21 Zhong YF, Zhang XW, Cai XF, Wang K, Chen YP, Deng YY: Puerarin attenuated early diabetic kidney injury through down-regulation of matrix metalloproteinase 9 in streptozotocin-induced diabetic rats. PLoS One 2014;9:e85690.

-22 Palsamy P, Subramanian S: Resveratrol protects diabetic kidney by attenuating hyperglycemia-mediated oxidative stress and renal inflammatory cytokines via Nrf2-Keap1 signaling. Biochim Biophys Acta 2011;1812:719-731.

23 Sun SF, Zhao TT, Zhang HJ, Huang XR, Zhang WK, Zhang L, Yan MH, Dong X, Wang H, Wen YM, Pan XP, Lan HY, Li P: Renoprotective effect of berberine on type 2 diabetic nephropathy in rats. Clin Exp Pharmacol Physiol 2015;42:662-670.

-24 Kitada M, Kume S, Imaizumi N, Koya D: Resveratrol improves oxidative stress and protects against diabetic nephropathy through normalization of Mn-SOD dysfunction in AMPK/SIRT1-independent pathway. Diabetes 2011;60:634-643.

-25 Chen KH, Hung CC, Hsu HH, Jing YH, Yang CW, Chen JK: Resveratrol ameliorates early diabetic nephropathy associated with suppression of augmented TGF- $\beta /$ smad and ERK1/2 signaling in streptozotocin-induced diabetic rats. Chem Biol Interact 2011;190:45-53.

26 Wu P, Yan Y, Ma LL, Hou BY, He YY, Zhang L, Niu ZR, Song JK, Pang XC, Yang XY, Du GH: Effects of the Nrf2 protein modulator salvianolic acid A alone or combined with metformin on diabetes-associated macrovascular and renal injury. J Biol Chem 2016;291:22288-22301.

27 Li L, Dong F, Xu D, Du L, Yan S, Hu H, Lobe CG, Yi F, Kapron CM, Liu J: Short-term, low-dose cadmium exposure induces hyperpermeability in human renal glomerular endothelial cells. J Appl Toxicol 2016;36:257-265.

28 Cajnko MM, Marušić M, Kisovec M, Rojko N, Benčina M, Caserman S, Anderluh G: Listeriolysin O affects the permeability of caco-2 monolayer in a pore-dependent and Ca2+-independent manner. PLoS One 2015;10:e0130471. 


\section{Cellular Physiology Cell Physiol Biochem 2017;44:2378-2394 \\ \begin{tabular}{l|l|l} 
and Biochemistry 10.1159/000486154 & $\begin{array}{l}\text { C) } 2017 \text { The Author(s). Published by S. Karger AG, Basel } \\
\text { www.karger.com/cpb }\end{array}$ \\
\hline
\end{tabular}}

Hou et al.: Renoprotective Effects of Salvianolic Acid A

29 Wang JP, Liu HX, Bo C, Qiang L, Huang XL, Wang LQ Guo XH, Huang QB: RhoA/ROCK-dependent moesin phosphorylation regulates AGE-induced endothelial cellular response. Cardiovasc Diabetol 2012;11:7.

30 Gallicchio MA, Bach LA: Uptake of advanced glycation end products by proximal tubule epithelial cells via macropinocytosis. Biochim Biophys Acta 2013;1833:2922-2932.

-31 Chilelli NC, Burlina S, Lapolla A: AGEs, rather than hyperglycemia, are responsible for microvascular complications in diabetes: a "glycoxidation-centric" point of view. Nutr Metab Carbiovasc Dis 2013;23:913919.

-32 Fleming T, Cuny J, Nawroth G, Djuric Z, Humpert PM, Zeier M, Bierhaus A, Nawroth PP: Is diabetes an acquired disorder of reactive glucose metabolites and their intermediates? Diabetologia 2012;55:11511155.

-33 Peppa M, Brem H, Cai W, Zhang JG, Basgen J, Li Z, Vlassara H, Uribarri J: Prevention and reversal of diabetic nephropathy in db/db mice treated with alagebrium (ALT-711). Am J Nephrol 2006;26:430-436.

-34 Freedman BI, Wuerth JP, Cartwright K, Bain RP, Dippe S, Hershon K, Mooradian AD, Spinowitz BS, Investigators AIS: Design and baseline characteristics for the aminoguanidine Clinical Trial in Overt Type 2 Diabetic Nephropathy (ACTION II). Control Clin Trials 1999;20:493-510.

-35 Lin J, Tang Y, Kang Q, Feng Y, Chen A: Curcumin inhibits gene expression of receptor for advanced glycation end-products (RAGE) in hepatic stellate cells in vitro by elevating PPAR $\gamma$ activity and attenuating oxidative stress. Br J Pharmacol 2012;166:2212-2227.

-36 Maricbilkan C, Flynn ER, Chade AR: Microvascular disease precedes the decline in renal function in the streptozotocin-induced diabetic rat. Am J Physiol-Renal Physiol 2012;302:F308-315.

37 Gavard J: Endothelial permeability and VE-cadherin. Cell Adhes Migr 2014;8:158-164.

-38 Guo X, Wang L, Chen B, Li Q Wang J, Zhao M, Wu W, Zhu P, Huang X, Huang Q: ERM protein moesin is phosphorylated by advanced glycation end products and modulates endothelial permeability. Am J PhysiolHeart Circul Physiol 2009;297:H238-246.

-39 Kuwabara A, Satoh M, Tomita N, Sasaki T, Kashihara N: Deterioration of glomerular endothelial surface layer induced by oxidative stress is implicated in altered permeability of macromolecules in Zucker fatty rats. Diabetologia 2010;53:2056-2065.

40 Veluthakal R, Kumar B, Mohammad G, Kowluru A, Kowluru RA: Tiam1-Rac1 axis promotes activation of p38 MAP kinase in the development of diabetic retinopathy: evidence for a requisite role for protein palmitoylation. Cell Physiol Biochem 2015;36:208-220.

-41 Sedeek M, Callera G, Montezano A, Gutsol A, Heitz F, Szyndralewiez C, Page P, Kennedy CR, Burns KD, Touyz RM, Hébert RL.: Critical role of Nox4-based NADPH oxidase in glucose-induced oxidative stress in the kidney: implications in type 2 diabetic nephropathy. Ajp Renal Physiology 2010;299:F1348-1358.

42 Zhang HA, Gao M, Zhang L, Zhao Y, Shi LL, Chen BN, Wang YH, Wang SB, Du GH: Salvianolic acid A protects human SH-SY5Y neuroblastoma cells against $\mathrm{H}_{2} \mathrm{O}_{2}$-induced injury by increasing stress tolerance ability. Biochem Biophys Res Commun 2012;421:479-483.

43 Cohen HY, Miller C, Bitterman KJ, Wall NR, Hekking B, Kessler B, Howitz KT, Gorospe M, de Cabo R, Sinclair DA: Calorie restriction promotes mammalian cell survival by inducing the SIRT1 deacetylase. Science 2004; 305:390-392.

44 Ding Y, Choi ME: Autophagy in diabetic nephropathy. J Endocrinol 2015;224:R15-30.

45 Kume S, Yamahara K, Yasuda M, Maegawa H, Koya D: Autophagy: emerging therapeutic target for diabetic nephropathy. Semin Nephrol 2014;34:9-16.

46 Liu WJ, Shen TT, Chen RH, Wu HL, Wang YJ, Deng JK, Chen QH, Pan Q, Huang FC, Tao JL, Liang D, Liu HF: Autophagy-lysosome pathway in renal tubular epithelial cells is disrupted by advanced glycation end products in diabetic nephropathy. J Biol Chem 2015;290:20499-20510.

47 Kitada M, Kume S, Takeda-Watanabe A, Kanasaki K, Koya D: Sirtuins and renal diseases: relationship with aging and diabetic nephropathy. Clin Sci (Lond) 2013;124:153-164.

48 Uribarri J, Cai W, Ramdas M, Goodman S, Pyzik R, Chen X, Zhu L, Striker GE, Vlassara H: Restriction of advanced glycation end products improves insulin resistance in human type 2 diabetes: Potential role of AGER1 and SIRT1. Diabetes Care 2011;34:1610-1616.

-49 Huang K, Huang J, Xie X, Wang S, Chen C, Shen X, Liu P, Huang H: Sirt1 resists advanced glycation end products-induced expressions of fibronectin and TGF- $\beta 1$ by activating the Nrf2/ARE pathway in glomerular mesangial cells. Free Radic Biol Med 2013;65:528-540. 